\title{
Reflets
}

Revue ontaroise d'intervention sociale et communautaire

\section{La sclérose en plaques, ses effets et les besoins spécifiques de ceux qu'elle afflige}

\section{Lise Nolet}

Volume 4, numéro 2, automne 1998

Personnes vivant avec une incapacité

URI : https://id.erudit.org/iderudit/026224ar

DOI : https://doi.org/10.7202/026224ar

Aller au sommaire du numéro

Éditeur(s)

Reflets : Revue ontaroise d'intervention sociale et communautaire

ISSN

1203-4576 (imprimé)

1712-8498 (numérique)

Découvrir la revue

Citer cet article

Nolet, L. (1998). La sclérose en plaques, ses effets et les besoins spécifiques de ceux qu'elle afflige. Reflets, 4(2), 144-150. https://doi.org/10.7202/026224ar

Tous droits réservés (C) Reflets : Revue ontaroise d'intervention sociale et communautaire, 1998
Ce document est protégé par la loi sur le droit d'auteur. L'utilisation des services d’Érudit (y compris la reproduction) est assujettie à sa politique d'utilisation que vous pouvez consulter en ligne.

https://apropos.erudit.org/fr/usagers/politique-dutilisation/ 


\title{
La sclérose en plaques, ses effets et les besoins spécifiques de ceux qu'elle afflige
}

\author{
Lise Nolet $^{1}$
}

Sudbury, avec la collaboration de Hellen Wagle et de Monique Cassidy

\section{La sclérose en plaque : une définition}

La sclérose en plaques (SP) est la maladie du système nerveux central la plus répandue chez les jeunes adultes au Canada. Elle s'attaque à la gaine de myéline des fibres nerveuses du système nerveux central, entraînant l'inflammation et souvent la détérioration de la myéline, par plaques. Ce phénomène nuit aux messages que reçoit le système nerveux et, par conséquent, l'influx nerveux ne se propage plus normalement. Les symptômes de cette maladie consistent en une vision affaiblie, un engourdissement, une perte d'équilibre, une fatigue extrême, des tremblements et même la paralysie ${ }^{2}$.

Les personnes atteintes de la sclérose en plaques éprouvent parfois des rémissions spontanées, lesquelles peuvent durer des mois, voire des années. Cependant, la sclérose en plaques est souvent progressive, c'est-à-dire qu'elle se caractérise par une série de poussées imprévisibles qui aggravent habituellement les malaises. La cause de ce mal, de même que son remède, ne sont pas encore connus. Toutefois, certains symptômes peuvent être atténués par des médicaments ou de la thérapie. 
La SP frappe généralement les personnes dont l'âge varie entre 20 et 40 ans, et presque deux fois plus de femmes que d'hommes en sont atteintes. Cette maladie se manifeste plus fréquemment dans les régions éloignées de l'équateur, telles que le Canada. Dans notre pays, des études épidémiologiques ont montré que le taux d'incidences de la SP figure parmi les plus élevés au monde.

\section{Les conséquences sociales et financières de la sclérose en plaques}

Comme la SP frappe au début de l'âge adulte, période où l'on commence à planifier sa carrière et sa vie familiale, il arrive assez souvent que les gens atteints de cette maladie doivent quitter leur emploi. Cette perte d'emploi crée un impact accru à long terme sur les familles et sur les individus affligés de la SP.

Les coûts sociaux et financiers sont élevés pour les personnes atteintes de la SP et pour leurs familles. En outre, quand le gouvernement ne réussit pas à subvenir aux besoins des gens, la société doit prendre le relais et assumer certaines responsabilités pécuniaires. Sans appareil, sans aide ménagère ou sans accès aux médicaments dispendieux, les personnes atteintes de la SP ne peuvent pas jouir d'une qualité de vie adéquate. Certaines personnes doivent même vivre au crochet de la société, c'est-à-dire recevoir de l'aide sociale ou un autre type d'assistance sociale.

\section{Les traitements disponibles}

Même s'il n'existe pas encore de remède pour enrayer la SP, les gens atteints de cette maladie peuvent avoir recours à des services et à des traitements qui peuvent alléger leurs symptômes. 
Dans le monde médical et pharmaceutique, les recherches avancent progressivement dans le domaine de la SP. Il existe aujourd'hui, d'après une large série d'essais cliniques, quatre traitements efficaces, capables de modifier l'évolution de la SP. À l'heure actuelle, les coûts de trois de ces traitements (Betaseron ${ }^{\text {md }}$, Rebif ${ }^{\text {nd }}$ ,Copaxone ${ }^{\mathrm{md}}$ ) sont remboursés par les régimes privés et collectifs d'assurance si la patiente ou le patient répond aux critères d'ordonnance. Les régimes de santé de certaines provinces, dont l'Ontario, remboursent les coûts de ces médicaments si la patiente ou le patient satisfait aux exigences établies par les programmes. Le Comité ontarien d'action sociale (COAS), appuyé en cela par le conseil d'administration de la division ontarienne de la SP, exerce une pression constante sur le gouvernement ontarien afin de s'assurer que les personnes répondant aux exigences établies par les régimes d'assurance maladie de la province accèdent aux traitements mis à leur disposition. De plus, en raison du coût élevé de ces médicaments, le COAS exerce une pression sur le gouvernement afin que les personnes bénéficiant de ces traitements soient remboursées dans un délai raisonnable.

En Ontario, il existe cinq cliniques spécialisées dans le traitement de la SP, dont une seulement offre des services en français. Et ces derniers sont limités à quelques services de base. On ne trouve aucune clinique dans le Nord de la province. Elles sont toutes situées dans le Sud et l'Est de l'Ontario, près des centres hospitaliers universitaires. Cela dit, le Ministère de la santé ne rembourse pas les frais de déplacement des gens du Nord qui désirent être traités à l'une ou l'autre de ces cliniques.

\section{La sclérose en plaques et les soins de longue durée}

La réforme des soins de longue durée, édictée par le gouvernement provincial à l'automne 1997, illustre clairement à quel point les jeunes personnes atteintes de maladies chroniques, comme la SP, sont souvent les grands oubliés des politiques en matière de santé ${ }^{3}$. En effet, cette réforme, comme bien d'autres, ne tient pas compte 
de la population de jeunes adultes qui, en raison d'un handicap ou d'une maladie chronique, devront recourir aux soins de longue durée. Cet oubli pourrait avoir de graves conséquences si le gouvernement néglige de prendre ses responsabilités et de répondre adéquatement aux besoins de cette population. Dans cette éventualité, on pourrait alors assister à une augmentation du niveau de stress et à la désintégration éventuelle du noyau familial. On pourrait aussi noter une augmentation du nombre de foyers pour les aînés, incapables de répondre aux besoins particuliers de ces patientes et patients qui ne relèvent pas de la gériatrie. Et enfin, on constatera le manque de ressources financières consacrées à la mise en vigueur d'une solution de remplacement plus convenable aux besoins de cette jeune population que les soins offerts en réseau institutionnel.

Le gouvernement se doit de prendre une position ferme face à la désinstitutionnalisation des soins de longue durée, surtout dans le cas des jeunes adultes. D'autres recherches doivent être faites afin d'explorer des solutions alternatives aux soins institutionnels pour les jeunes adultes ayant une maladie ou un handicap chronique ou progressif, tel que la sclérose en plaques. Déjà, certains services semblent prometteurs comme les résidences communautaires, un programme de soins quotidiens de santé pour les adultes et des services de répit aux aidantes ou aux aidants naturels.

En matière de soins de longue durée, les besoins des personnes vivant avec la SP sont très différents, à cause des rémissions et des rechutes spontanées qui peuvent durer des semaines, des mois voire même, des années ${ }^{4}$. La façon dont la maladie évolue pose donc un grand défi dans les soins prodigués en vue d'atténuer les symptômes. Par exemple, une personne atteinte de la SP peut avoir besoin de services sporadiquement au cours d'une année.Toutefois, lorsque survient une crise ou une rechute, elle doit être en mesure d'en bénéficier immédiatement ${ }^{5}$. 


\section{La Société de la sclérose en plaques}

Fondée en 1962, la division ontarienne de la Société canadienne de la sclérose en plaques regroupe 12000 membres. Cet organisme est le seul qui appuie le développement de la recherche et des services qui permettent de répondre aux besoins des gens atteints de sclérose en plaques et à ceux de leurs familles.

La mission de la Société canadienne de la SP est d'être un chef de file pour enrayer la SP et pour redonner le pouvoir aux gens qui sont aux prises avec la maladie, afin de les aider à améliorer leur qualité de vie.

La division ontarienne de la Société canadienne de la SP peut atteindre ce but, grâce à l'aide de bénévoles qui œuvrent au sein de divers comités. Parmi ces comités, le COAS joue un rôle très important. Ce comité, formé de gens atteints de la maladie ou de leurs proches, est en quelque sorte les yeux et les oreilles de la division ontarienne de la Société canadienne de la SP. Le COAS aborde les dossiers politiques ayant un impact sur la vie des personnes atteintes de la SP. Il arrive aussi que le COAS propose une stratégie de revendication sur le dossier en question. En outre, le COAS appuie non seulement les individus atteints de la SP, mais aussi les chapitres locaux de la Société de la SP dans leurs luttes d'action sociale. Le COAS peut avoir une influence sur les politiques gouvernementales avant même que celles-ci ne deviennent des programmes ou des services officiels. Le COAS joue aussi un rôle de sensibilisation important auprès du gouvernement et du grand public. Bien que la maladie de la SP ait été découverte depuis près de cent ans, elle demeure encore aujourd'hui une énigme.

\section{Conclusion}

Il reste encore plusieurs défis à relever au sujet de la SP. La réforme 
des soins de longue durée n'est qu'un des nombreux exemples qui permettra d'assurer une qualité de vie satisfaisante chez les gens atteints de la SP. Ces derniers éprouvent plusieurs autres besoins: l'accès à l'éducation ou à la formation en emploi dans le cas des personnes dont la maladie les force à se réorienter dans une autre profession, l'accessibilité aux édifices et aux transports en commun, l'accès à de l'aide ménagère ou encore, l'accès aux appareils techniques (marchettes, cannes, fauteuils roulants, etc.) facilitant la vie de ceux et celles atteints de la maladie.

Munies d'une meilleure compréhension de la sclérose en plaques et de son évolution, les personnes atteintes de cette maladie pourraient enfin espérer recevoir plus facilement l'aide qui leur permettrait de jouir pleinement de la vie.

\section{Bibliographie}

MULTIPLE SCLEROSIS SOCIETY OF CANADA, ONTARIO DIVISION (1997). Healthy Future?: Addressing the needs of younger populations with multiple sclerosis and other chronic illnesses in a restructured health care system, Toronto.

\section{Notes}

1. Lise Nolet est présidente du Comité ontarien de l'action sociale, Société canadienne de la sclérose en plaques et viceprésidente au Conseil d'administration de la division ontarienne de la Société canadienne de la sclérose en plaques. Cet article a été écrit avec la collaboration de Helen Wagle, responsable de l'action sociale, Société canadienne de la sclérose en plaques et de Monique Cassidy, membre du Comité ontarien de l'action sociale, Société canadienne de la sclérose en plaques. Les opinions dans ce texte sont celles de l'auteure et non celles de la Société canadienne de la sclérose en plaques.

2. Cette définition provient du document intitulé «Renseignements 1997 sur la Société canadienne de la sclérose en plaques», 1997, Toronto, Société canadienne de la sclérose en plaques. 
3. Cette section est extraite du document de Multiple Sclerosis Society of Canada, Ontario Division,Toronto, September 1997.

4. Frankel, D., "Long-Term Care Issues in Multiple Sclerosis», Rehabilitation Literature, vol. 45, no 9, 282-285

5. Nolet, L. (1994). «Some Concerns of Women in Long-Term Health Care Reform", Submission to the Standing Committee on Social Development of the Legislative Assembly of Ontario, Ottawa, September. 